\title{
Mean-field description of a dynamical collapse of a fermionic condensate in a trapped boson-fermion mixture
}

\author{
Sadhan K. Adhikari \\ Instituto de Física Teórica, Universidade Estadual Paulista, 01.405-900 São Paulo, São Paulo, Brazil \\ (Received 24 April 2004; revised manuscript received 1 July 2004; published 19 October 2004)
}

\begin{abstract}
We suggest a time-dependent dynamical mean-field-hydrodynamic model for the collapse of a trapped boson-fermion condensate and perform numerical simulation based on it to understand some aspects of the experiment by Modugno et al. [Science 297, 2240 (2002)] on the collapse of the fermionic condensate in the ${ }^{40} \mathrm{~K}-{ }^{87} \mathrm{Rb}$ mixture. We show that the mean-field model explains the formation of a stationary boson-fermion condensate at zero temperature with relative sizes compatible with experiment. This model is also found to yield a faithful representation of the collapse dynamics in qualitative agreement with experiment. In particular we consider the collapse of the fermionic condensate associated with (a) an increase of the number of bosonic atoms as in the experiment and (b) an increase of the attractive boson-fermion interaction using a Feshbach resonance. Suggestion for experiments of fermionic collapse using a Feshbach resonance is made.
\end{abstract}

DOI: 10.1103/PhysRevA.70.043617

PACS number(s): $03.75 . \mathrm{Nt}$

\section{INTRODUCTION}

Recent successful observation of condensed bosonfermion mixtures of trapped alkali-metal atoms by different experimental groups [1-4] has initiated the intensive experimental studies of different novel phenomena [5-7]. Among these experiments there have been studies of condensate of two components of ${ }^{40} \mathrm{~K}$ [1] and ${ }^{6} \mathrm{Li}$ [2] atoms. Condensation of boson-fermion mixtures ${ }^{6,7} \mathrm{Li}[3],{ }^{23} \mathrm{Na}-{ }^{6} \mathrm{Li}$ [4], and ${ }^{87} \mathrm{Rb}-$ ${ }^{40} \mathrm{~K}[5,6]$ have also been reported. The collapse of fermionic condensate in a boson-fermion mixture ${ }^{87} \mathrm{Rb}-{ }^{40} \mathrm{~K}$ has been observed and studied by Modugno et al. [5]. In this paper we consider the collapse dynamics of fermions in a bosonfermion mixture using a coupled time-dependent mean-fieldhydrodynamic model. The bosonic part is treated by the mean-field Gross-Pitaevskii (GP) equation [8] and the fermionic part is treated by a hydrodynamic model. The present mean-field-hydrodynamic model could be considered to be a time-dependent extension of a time-independent model suggested for the stationary equilibrium states by Capuzzi et al. [9].

Because of the Pauli exclusion principle the fermions in spin parallel states in the condensate experience a strong repulsion. This is the dominating interaction at short distances and avoids the collapse of a fermionic condensate. In contrast an attractive bosonic condensate larger than a critical size is not dynamically stable [10]. However, if such a bosonic condensate is "prepared" or somehow made to exist it experiences a dramatic collapse and explodes emitting atoms leading to a condensate of small size with a high central density. Under high pressure three-body recombination takes place with the emission of energy leading to explosion and loss of atoms. The possibility of collapse was first suggested and observed in ${ }^{7} \mathrm{Li}$ atoms [10]. A dynamical study of controlled collapse and explosion has been performed by Donley et al. [11] on an attractive ${ }^{85} \mathrm{Rb}$ bosonic condensate, where they manipulated the interatomic interaction by changing the external magnetic field exploiting a nearby Feshbach resonance [12]. In the vicinity of a Feshbach resonance the atomic scattering length $a$ can be varied over a huge range by adjusting an external magnetic field, thus transforming a repulsive bosonic condensate to a highly attractive one. There have been many theoretical $[13,14]$ studies to describe different features of the experiment by Donley et al. [11].

In the classic study of a trapped boson-fermion mixture Modugno et al. [5] simulated a situation of a strong effective interaction between fermions via a strongly attractive bosonfermion interaction which may play an important role in the collapse of the fermionic condensate. The presence of a strong attractive interaction between bosons and fermions can induce an effective attraction between the fermions [15]. If this overall effective attraction among the fermions can overcome the Fermi repulsive pressure there is a possibility of the collapse of a fermionic condensate in a boson-fermion mixture. Modugno et al. [5] showed in an experiment with ${ }^{87} \mathrm{Rb}-{ }^{40} \mathrm{~K}$ mixture that it is indeed the case and produced some results of the collapsing dynamics of this system which we would like to understand using a mean-fieldhydrodynamic model.

Previously, in addition to the study of the collapse of a purely bosonic condensate [13,14], we also investigated [16-18] the collapse dynamics in a two-species bosonic condensate initiated by an attraction between interspecies bosons using the mean-field coupled GP equation [8]. It was found that even when the intra-species interaction is repulsive one can have a collapse in one or two species due to the interspecies attraction which simulates an effective intraspecies attraction. The situation is qualitatively similar in the present boson-fermion mixture. The boson-fermion attraction in a boson-fermion mixture can induce an attraction in the fermionic system leading to collapse. However, there is no coupled GP equation in the case of boson-fermion mixture which complicates a theoretical description. Instead, in this paper we use a time-dependent version of a recently suggested equations of generalized hydrodynamics [9]. These equations allow us to span the entire range of boson-fermion interaction as in the coupled GP equation for bosons [16].

The equilibrium properties and the phase diagram of a boson-fermion mixture have been studied by several authors 
[19] to obtain good agreement with experiment using a mean-field-type description [20]. All of them employed a time-independent formulation. Here, the study of nonequilibrium properties of fermionic collapse using a time-dependent formulation is considered. Our findings are in agreement with the experiment by Modugno et al. [5].

In Sec. II we present our time-dependent mean-field model. This consists of a set of coupled partial differential equations involving the bosonic and fermionic condensate wave functions. We introduce an appropriate loss term due to three-body boson-fermion recombination. In Sec. III we present our results for stationary boson-fermion wave functions as well as fermion collapse initiated by a growth in boson number and boson-fermion interaction. Our stationary results are consistent with experiment [5] and other numerical studies $[9,19,20]$. The present study also yields a faithful representation of the collapse dynamics as observed experimentally [5]. Finally, a summary of our findings is given in Sec. IV.

\section{NONLINEAR MEAN-FIELD-HYDRODYNAMIC MODEL}

The time-dependent Bose-Einstein condensate wave function $\Psi(\mathbf{r} ; t)$ at position $\mathbf{r}$ and time $t$ allowing for atomic loss may be described by the following mean-field nonlinear GP equation [8]

$$
\left[-i \hbar \frac{\partial}{\partial t}-\frac{\hbar^{2} \nabla^{2}}{2 m_{B}}+V_{B}(\mathbf{r})+g_{B B} n_{B}\right] \Psi_{B}(\mathbf{r} ; t)=0,
$$

with normalization $\int d \mathbf{r}\left|\Psi_{B}(\mathbf{r} ; t)\right|^{2}=N_{B}$. Here $m_{B}$ is the mass and $N_{B}$ the number of bosonic atoms in the condensate, $n_{B}$ $\equiv\left|\Psi_{B}(\mathbf{r} ; t)\right|^{2}$ is the boson probability density, $g_{B B}$ $=4 \pi \hbar^{2} a_{B B} / m_{B}$ the strength of interatomic interaction, with $a_{B B}$ the scattering length. The trap potential with spherical symmetry may be written as $V_{B}(\mathbf{r})=1 / 2 m_{B} \omega_{B}^{2} r^{2}$, where $\omega_{B}$ is the angular frequency. The probability density of an isolated fermionic condensate $n_{F} \equiv\left|\Psi_{F}(\mathbf{r})\right|^{2}$ is given by

$$
n_{F}=\frac{\left[\epsilon_{F}-V_{F}(\mathbf{r})\right]^{3 / 2}}{A^{3 / 2}},
$$

where $\Psi_{F}(\mathbf{r})$ is the condensate wave function, $A$ $=\hbar^{2}\left(6 \pi^{2}\right)^{2 / 3} /\left(2 m_{F}\right), \epsilon_{F}$ is the Fermi energy, and $m_{F}$ is the fermionic mass. The spherical trap is given by $V_{F}(\mathbf{r})$ $=\frac{1}{2} m_{F} \omega_{F}^{2} r^{2}$. The number of fermionic atoms $N_{F}$ is given by the normalization $\int d \mathbf{r}\left|\Psi_{F}(\mathbf{r})\right|^{2}=N_{F}$.

However, it has been suggested that if there is an interaction between the fermions and bosons, Eqs. (2.1) and (2.2) get modified to [20]

$$
\left[-i \hbar \frac{\partial}{\partial t}-\frac{\hbar^{2} \nabla^{2}}{2 m_{B}}+V_{B}(\mathbf{r})+g_{B B} n_{B}+g_{B F} n_{F}\right] \Psi_{B}(\mathbf{r} ; t)=0
$$

and

$$
n_{F}=\frac{\left(\epsilon_{F}-V_{F}-g_{B F} n_{B}\right)^{3 / 2}}{A^{3 / 2}},
$$

where $g_{B F}=2 \pi \hbar^{2} a_{B F} / m_{R}$ with the boson-fermion reduced mass $m_{R}=m_{B} m_{F} /\left(m_{B}+m_{F}\right)$ where $m_{F}$ is the mass of fermionic atoms. The interaction between fermions in spin polarized state is highly suppressed due to Pauli exclusion principle and has been neglected in Eqs. (2.3) and (2.4) and will be neglected throughout this paper. Also, we shall always assume boson-fermion attraction $\left(a_{B F}<0\right)$ and boson-boson repulsion $\left(a_{B B}>0\right)$.

Capuzzi et al. [9] developed a set of time-independent equations for boson-fermion condensate from a consideration of hydrodynamic motion of two condensed fluids, one bosonic and another fermionic, in a spherical trap at zero temperature. The interaction between bosons and between bosons and fermions are described by contact potentials parametrized by coupling constants $g_{B B}$ and $g_{B F}$ defined above. They derived the following mean-field energy functional for the system [9]

$$
\begin{aligned}
{[E]=} & \int d \mathbf{r}\left(\frac{\hbar^{2}\left|\nabla \Psi_{B}\right|^{2}}{2 m_{B}}+V_{B}\left|\Psi_{B}\right|^{2}+\frac{1}{2} g_{B B}\left|\Psi_{B}\right|^{4}\right) \\
& +\int d \mathbf{r}\left(\frac{\hbar^{2}\left|\nabla \Psi_{F}\right|^{2}}{6 m_{F}}+V_{F}\left|\Psi_{F}\right|^{2}+\frac{3}{5} A\left|\Psi_{F}\right|^{10 / 3}\right) \\
& +g_{B F} \int d \mathbf{r}\left|\Psi_{F}\right|^{2}\left|\Psi_{B}\right|^{2} .
\end{aligned}
$$

The first integral on the right-hand side of Eq. (2.5) is the Gross-Pitaevskii energy functional of the bosons and is related to the (nonlinear) Schrödinger equation [8]. However, the second integral, although bears a resemblance with the first, is derived from the hydrodynamic equation of motion of the fermions and is not related to a Schrödinger-like equation [9]. Hence, the derivative term in the second integral has a different mass factor $6 m_{F}$ and not the conventional Schrödinger mass factor $2 m_{B}$ as in the first integral. Finally, the last integral corresponds to an interaction between bosons and fermions.

Equation (2.5) corresponds to the following Lagrangian density:

$$
\begin{aligned}
\mathcal{L}= & \frac{i}{2} \hbar \sum_{i=B, F}\left(\Psi_{i} \frac{\partial \Psi_{i}^{*}}{\partial t}-\Psi_{i}^{*} \frac{\partial \Psi_{i}}{\partial t}\right)+\left(\frac{\hbar^{2}\left|\nabla \Psi_{B}\right|^{2}}{2 m_{B}}+V_{B}\left|\Psi_{B}\right|^{2}\right. \\
& \left.+\frac{1}{2} g_{B B}\left|\Psi_{B}\right|^{4}\right)+\left(\frac{\hbar^{2}\left|\nabla \Psi_{F}\right|^{2}}{6 m_{F}}+V_{F}\left|\Psi_{F}\right|^{2}+\frac{3}{5} A\left|\Psi_{F}\right|^{10 / 3}\right) \\
& +g_{B F}\left|\Psi_{F}\right|^{2}\left|\Psi_{B}\right|^{2} .
\end{aligned}
$$

The mean-field dynamical equations for the system are just the usual Euler-Lagrange equations

$$
\frac{d}{d t} \frac{\partial \mathcal{L}}{\partial \frac{\partial \Psi_{i}^{*}}{\partial t}}+\sum_{k=1}^{3} \frac{d}{d x_{k}} \frac{\partial \mathcal{L}}{\partial \frac{\partial \Psi_{i}^{*}}{\partial x_{k}}}=\frac{\partial \mathcal{L}}{\partial \Psi_{i}^{*}},
$$

where $x_{k}, k=1,2,3$ are the three space components, $i=B$ corresponds to the bosonic wave function and $i=F$ corresponds 
to the fermionic wave function. With the Lagrangian density (2.6) these equations of motion become

$$
\begin{aligned}
& {\left[-i \hbar \frac{\partial}{\partial t}-\frac{\hbar^{2} \nabla^{2}}{2 m_{B}}+V_{B}(\mathbf{r})+g_{B B} n_{B}+g_{B F} n_{F}\right] \Psi_{B}(\mathbf{r} ; t)=0} \\
& {\left[-i \hbar \frac{\partial}{\partial t}-\frac{\hbar^{2} \nabla^{2}}{6 m_{F}}+V_{F}(\mathbf{r})+A n_{F}^{2 / 3}+g_{B F} n_{B}\right] \Psi_{F}(\mathbf{r} ; t)=0 .}
\end{aligned}
$$

The normalization of the wave functions are given by $\int d \mathbf{r}\left|\Psi_{B}(\mathbf{r} ; t)\right|^{2}=N_{B}$ and $\int d \mathbf{r}\left|\Psi_{F}(\mathbf{r} ; t)\right|^{2}=N_{F}$. It is understood that Eq. (2.4) results as a consequence of Thomas-Fermi-type approximation to Eq. (2.9), when the kinetic energy term in the latter (equation) is neglected at low temperatures.

Equations (2.8) and (2.9) are the desired time-dependent mean-field-hydrodynamic equations. We shall use these equations for the study of the collapse of the fermionic atoms in a coupled boson-fermion mixture. If $g_{B F}$ were zero, both the bosonic and fermionic condensates would have been stable. An attractive or negative $g_{B F}$ can make one or both systems to become highly attractive and lead to collapse. Similar effect was found before in the study of two coupled bosonic condensates [16]. In that study it was found that even if the interaction among atoms of the same species is repulsive an attractive interaction among atoms of two different species can cause one or both components of the coupled system to collapse. Similarly, in a coupled atomicmolecular condensate a strong attraction between atoms and molecules can cause one or both of atomic and molecular condensates to collapse [17].

To study the phenomenon of collapse we have to add the proper mechanism for atom loss in Eqs. (2.8) and (2.9). The attractive interaction between a bosonic atom $B$ and a fermionic atom $F$ leads to the following three-body recombination process to form a molecule $(B F)$ composed of a bosonic and fermionic atom, which is responsible for the loss of atoms [5] :

$$
B+B+F \rightarrow(B F)+B .
$$

As the fermions are in a spin-polarized state, due to Pauli principle two-fermion molecules cannot be formed. Consequently, femionic atoms could only be lost in the presence of bosons and the loss rate scales quadratically with bosonic density and is independent of fermion number $N_{F}$ [5]. In addition to reaction (2.10), there is also the possibility of the formation of a two-boson molecule $(B B)$ via the reaction

$$
B+B+B \rightarrow(B B)+B .
$$

However, for a low-density repulsive bosonic condensate as in the experiment on boson-fermion mixture [5], the rate of loss of bosons due to reaction (2.11) will presumably be small [21]. Moreover, reaction (2.11) has no influence on the collapse of the fermionic condensate. As the primary motivation of this paper is to investigate the collapse of the fermionic condensate, we neglect reaction (2.11) in our study.

The recombination reaction (2.10) adds an extra term in Lagrangian density (2.6) of the form $-i \hbar K_{3}\left|\Psi_{F}\right|^{2}\left|\Psi_{B}\right|^{4} / 2$ proportional to the product of fermion density and the square of boson density, where $K_{3}$ is the loss rate. In the presence of only the bosonic atoms the contribution to the Lagrangian density for the recombination reaction (2.11) is the usual three-body recombination term $-i \hbar K_{3}|\Psi|^{6} / 2[14,21]$ proportional to the cube of boson density. After proper symmetrization, with the use of the loss term $-i \hbar K_{3}\left|\Psi_{F}\right|^{2}\left|\Psi_{B}\right|^{4} / 2$ in Eq. (2.6), Eqs. (2.8) and (2.9) become

$$
\begin{gathered}
{\left[-i \hbar \frac{\partial}{\partial t}-\frac{\hbar^{2} \nabla^{2}}{2 m_{B}}+V_{B}(\mathbf{r})+g_{B B} n_{B}+g_{B F} n_{F}-i \hbar K_{3} n_{B} n_{F}\right]} \\
\times \Psi_{B}(\mathbf{r} ; t)=0, \\
{\left[-i \hbar \frac{\partial}{\partial t}-\frac{\hbar^{2} \nabla^{2}}{6 m_{F}}+V_{F}(\mathbf{r})+A n_{F}^{2 / 3}+g_{B F} n_{B}-i \hbar K_{3} n_{B}^{2}\right]} \\
\times \Psi_{F}(\mathbf{r} ; t)=0 .
\end{gathered}
$$

In the spherically symmetric state of zero angular momentum the wave function has the form $\Psi(\mathbf{r} ; t)=\psi(r ; t)$. Of the experimental condensation of boson-fermion mixtures the two pioneering possibilities were the mixture of ${ }^{6} \mathrm{Li}$ (fermion) and ${ }^{23} \mathrm{Na}$ (boson) [4], and the mixture of ${ }^{40} \mathrm{~K}$ (fermion) and ${ }^{87} \mathrm{Rb}$ (boson) [5]. The actual experiment on the collapse of fermions [5] was performed in the ${ }^{40} \mathrm{~K}-{ }^{87} \mathrm{Rb}$ system with an axially symmetric trap. In this paper we shall consider the effect of the variation of the boson-fermion scattering length $a_{B F}$ on the collapse. For that purpose one requires not only an accurate value of $K_{3}$ but a variation of $K_{3}$ with $a_{B F}$. As the dynamics of collapse is sensitive to this unknown variation of $K_{3}$, we are not in a position to produce the (quantitative) dynamics of collapse in the realistic (experimental) situation. Instead, in this paper we consider a spherically symmetric model to see if this model can describe the essentials of the observed collapse dynamics [5]. We also present results on central probability density of the condensate to confirm the collapse.

As we shall not be interested in a particular bosonfermion system in this paper, but will be concerned with the collapse of the fermionic condensate in general we take in the rest of this paper $m_{B}=3 m_{F}=m(\mathrm{Rb})$, whence $m_{R}=3 m_{F} / 4$. In the above two experimental situations [4,5] $m_{B} \approx 3 m_{F}$. Also, we take $V_{B}(\mathbf{r})=V_{F}(\mathbf{r})=m_{B} \omega_{B}^{2} r^{2} / 2$ which corresponds to a reduction of $\omega_{B}$ by a factor $\sqrt{m_{B} / m_{F}}$ as in the study by Modugno et al. [20]. These two assumptions give a simpler analytical form of the final equations we derive eliminating factors of masses and frequencies without any consequence to our qualitative study. Now transforming to dimensionless variables defined by $x=\sqrt{2} r / l, \tau=t \omega, l \equiv \sqrt{\hbar /\left(m_{B} \omega_{B}\right)}$ and 


$$
\frac{\varphi_{i}(x ; \tau)}{x}=\sqrt{\frac{4 \pi l^{3}}{N_{i} \sqrt{8}}} \psi_{i}(r ; t),
$$

$i=B, F$, we get

$$
\begin{aligned}
& {\left[-i \frac{\partial}{\partial \tau}-\frac{\partial^{2}}{\partial x^{2}}+\frac{x^{2}}{4}+2 \sqrt{2} \mathcal{N}_{B B}\left|\frac{\varphi_{B}}{x}\right|^{2}+4 \sqrt{2} \mathcal{N}_{B F}\left|\frac{\varphi_{F}}{x}\right|^{2}\right.} \\
& \left.-i \xi_{B} \mathcal{N}_{B B} \mathcal{N}_{B F}\left|\frac{\varphi_{B}}{x}\right|^{2}\left|\frac{\varphi_{F}}{x}\right|^{2}\right] \times \varphi_{B}(x ; \tau)=0, \\
& {\left[-i \frac{\partial}{\partial \tau}-\frac{\partial^{2}}{\partial x^{2}}+\frac{x^{2}}{4}+3\left(\frac{3 \pi N_{F}}{2}\right)^{2 / 3}\left|\frac{\varphi_{F}}{x}\right|^{4 / 3}+4 \sqrt{2} \mathcal{N}_{F B}\left|\frac{\varphi_{B}}{x}\right|^{2}\right.} \\
& \left.-i \xi_{F} \mathcal{N}_{B B}^{2}\left|\frac{\varphi_{B}}{x}\right|^{4}\right] \times \varphi_{F}(x ; \tau)=0,
\end{aligned}
$$

where $\mathcal{N}_{B B}=N_{B} a_{B B} / l, \quad \mathcal{N}_{B F}=N_{F} a_{B F} / l, \quad \mathcal{N}_{F B}=N_{B} a_{B F} / l, \quad \xi_{B}$ $=K_{3} /\left(2 \pi^{2} a_{B B} a_{B F} l^{4} \omega_{B}\right)$, and $\xi_{F}=K_{3} /\left(2 \pi^{2} a_{B B}^{2} l^{4} \omega_{B}\right)$. In the nonabsorptive case $\xi_{F}=\xi_{B}=0$, the normalization of the wavefunction components is given by $\int_{0}^{\infty} d x\left|\varphi_{i}(x ; \tau)\right|^{2}=1, i=B, F$. In the absorptive case $\xi_{F} \neq 0$ and $\xi_{B} \neq 0$, the normalization reduces with time due to loss of atoms.

\section{NUMERICAL RESULT}

We solve the coupled mean-field-hydrodynamic eqs. (2.15) and (2.16) numerically using a time-iteration method based on the Crank-Nicholson discretization scheme elaborated in Ref. 22. We discretize the GP equation using time step 0.00025 and space step 0.05 spanning $x$ from 0 to 25 . This domain of space was sufficient to encompass the whole condensate wave function during collapse and expansion.

First we solve Eqs. (2.15) and (2.16) with $\xi_{B}=\xi_{F}=0$. This will allow us to find a stable bound state of boson-fermion mixture. After some experimentation we take in our calculation $a_{B B}=5 \mathrm{~nm}, \quad a_{B F}=-12.5 \mathrm{~nm}, \quad \omega_{B}=2 \pi \times 100 \mathrm{~Hz}, \quad N_{B}$ $=4800$, and $N_{F}=1200$, so that $l \approx 1 \mu \mathrm{m}, a_{B B} / l=0.005$, $a_{B F} / l=-0.0125, \mathcal{N}_{B B}=24, \mathcal{N}_{B F}=-15$, and $\mathcal{N}_{F B}=-60$. These values of parameters are similar to those employed in experiments. The dimensionless unit of time corresponds to $\omega_{B}^{-1}$ $\approx 1.6 \mathrm{~ms}$, and dimensionless unit of length corresponds to $l / \sqrt{2} \approx 0.7 \mu \mathrm{m}$.

Now we consider the collapse of fermions initiated by a sudden jump in the boson-fermion scattering length from $a_{B F}=-12.5 \mathrm{~nm}$ to $-37.5 \mathrm{~nm}$ which can be implemented near a boson-fermion Feshbach resonance. Such boson-fermion Feshbach resonances between ${ }^{23} \mathrm{Na}$ (boson) and ${ }^{6} \mathrm{Li}$ (fermion) atoms [23] and between ${ }^{87} \mathrm{Rb}$ (boson) and ${ }^{40} \mathrm{~K}$ (fermion) atoms [24] have been experimentally observed. These resonances should enable experimental control of the interspecies interactions [24] and hence can be used to increase the attractive force between bosons and fermions which in turn increases the attractive nonlinearities $4 \sqrt{2} \mathcal{N}_{B F}$ and $4 \sqrt{2} \mathcal{N}_{F B}$ in Eqs. (2.15) and (2.16). If these attractive nonlin-

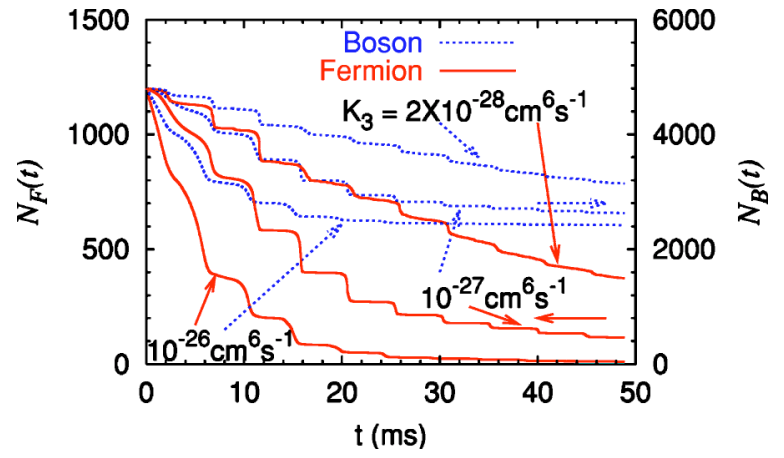

FIG. 1. (Color online) The evolution of the numbers of bosons $N_{B}(t)$ and fermions $N_{F}(t)$ during collapse initiated by a jump in boson-fermion scattering length $a_{B F}$ from -12.5 to $-37.5 \mathrm{~nm}$ for $K_{3}=2 \times 10^{-28}, 10^{-27}$, and $10^{-26} \mathrm{~cm}^{6} \mathrm{~s}^{-1}$. The three upper (blue, dotted) curves refer to number of bosons and three lower (red, solid) curves refer to number of fermions. The curves are labeled by their respective $K_{3}$ values.

ear terms are larger than the repulsive nonlinearities in these equations it is possible to have collapse of fermions or bosons or both. In order to have collapse, the effective nonlinearities in these equations should be attractive.

Due to the imaginary terms in Eqs. (2.15) and (2.16) the numbers of bosons and fermions decay with time. When the net nonlinear attraction in these equations is small there is smooth and steady decay of number of atoms. However, when the net nonlinear attraction is gradually increased the steady decay of number of atoms develops into a violent decay called collapse. When this happens the condensate loses a significant fraction of atoms in a small interval of time (milliseconds) after which a remnant condensate with a reasonably constant number of atoms is formed. Also, during and immediately after collapse, the wave function of the condensate becomes very unsmooth and spiky in nature as opposed to a reasonably smooth wave function in the case of a steady decay.

We study the the evolution of the boson and fermion numbers in the condensate from time $t=0$ to $t=50 \mathrm{~ms}$ after the sudden jump in the scattering length from $a_{B F}=-12.5$ to $-37.5 \mathrm{~nm}$ at time $t=0$. The evolution of boson and fermion numbers depends on the value of the loss rate $K_{3}$. We study the sensitivity of the result on $K_{3}$ by performing the calculation for different loss rates. In Fig. 1 we plot the evolution of the boson and fermion numbers for different loss rates $K_{3}$ $=2 \times 10^{-28}, 10^{-27}$, and $10^{-26} \mathrm{~cm}^{6} \mathrm{~s}^{-1}$. With the increase of $K_{3}$ the rate of decay increases, although the results for different $K_{3}$ are qualitatively similar. We see in Fig. 1 that in all cases both the number of bosons and fermions decay rapidly and attain an approximately constant (remnant) number in less than $50 \mathrm{~ms}$. The panorama is similar to the collapse in attractive bosonic condensate studied experimentally by Donley et al. [11], where also a cold remnant bosonic condensate is formed at the end of the collapse. In the recombination process (2.10) two bosonic and one fermionic atoms are lost. In Fig. 1 we find that during the collapse about 2000 bosonic atoms and 1000 fermionic atoms are lost.

Experimentally, Modugno et al. measured the following loss rate $K_{3}=2(1) \times 10^{-27} \mathrm{~cm}^{6} \mathrm{~s}^{-1}$ for the ${ }^{40} \mathrm{~K}-{ }^{87} \mathrm{Rb}$ system 


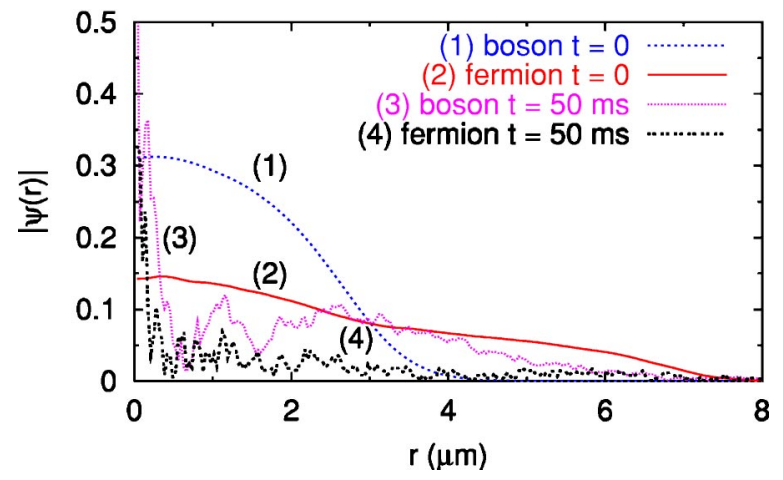

FIG. 2. (Color online) The initial $(t=0)$ and final $(t=50 \mathrm{~ms})$ fermion and boson wave functions in arbitrary units before and after collapse initiated by a jump in boson-fermion scattering length $a_{B F}$ from -12.5 to $-37.5 \mathrm{~nm}$.

[5]. In the remainder of this paper we shall use the value $K_{3}=10^{-27} \mathrm{~cm}^{6} \mathrm{~s}^{-1}$ for all densities and all values of scattering lengths. This value of $K_{3}$ gives a loss rate during collapse compatible with experiment [5], where a significant fraction of fermions are lost in an interval of time $\Delta<50 \mathrm{~ms}$. We further examined the wave function of the bosons and fermions to determine whether the decay in Fig. 1 really corresponds to a collapse and not to a slow evaporation due to the imaginary terms in Eqs. (2.15) and (2.16).

In Fig. 2 we plot the profiles of the bosonic and fermionic wave functions in arbitrary units at times $t=0$ and $t=50 \mathrm{~ms}$. A close look at Fig. 2 reveals that before collapse at $t=0$ the bosonic and fermionic wave functions are smooth and the fermionic wave function extends over a larger distance than the bosonic wave function. This is due to the large repulsion between the fermions in the spin polarized state due to the Pauli exclusion principle. Hence the bosonic condensate lies well inside the fermionic condensate. This was noted in experiment [5], as well as in previous theoretical studies $[9,19,20]$. The wave functions after collapse have an entirely different profile. As expected the wave functions are highly peaked in the central $(r=0)$ region and develop spikes. However, they extend over a large distance too. The final spiky wave function indicates the collapse in contrast to a smooth final wave function corresponding to a steady loss of atoms. The collapse is a quick process lasting at most a few tens of milliseconds when a significant fraction of atoms are lost. For example, in Fig. 1 for $K_{3}=10^{-27} \mathrm{~cm}^{6} \mathrm{~s}^{-1}$, the collapse lasts for the first $25 \mathrm{~ms}$ when most of the atoms are lost. After this interval the rate of loss of atoms is reduced and remnant bosonic and fermionic condensates with a roughly constant number of atoms are formed. The wave function after the collapse of a purely bosonic condensate also exhibits a similar behavior [14].

To confirm further the collapse in Figs. 1 and 2 for $K_{3}$ $=10^{-27} \mathrm{~cm}^{6} \mathrm{~s}^{-1}$, we plot in Fig. 3 the evolution of the central probability density of (a) the bosonic $\left[N_{B}\left|\psi_{B}(0, t)\right|^{2}\right]$ and (b) fermionic $\left[N_{F}\left|\psi_{F}(0, t)\right|^{2}\right]$ components in arbitrary units during collapse. We note a very strong fluctuation of the central density reminiscent of collapse both in the bosonic and fermionic components. Similar variations are common to the collapse of a purely bosonic condensate [14]. Such strong
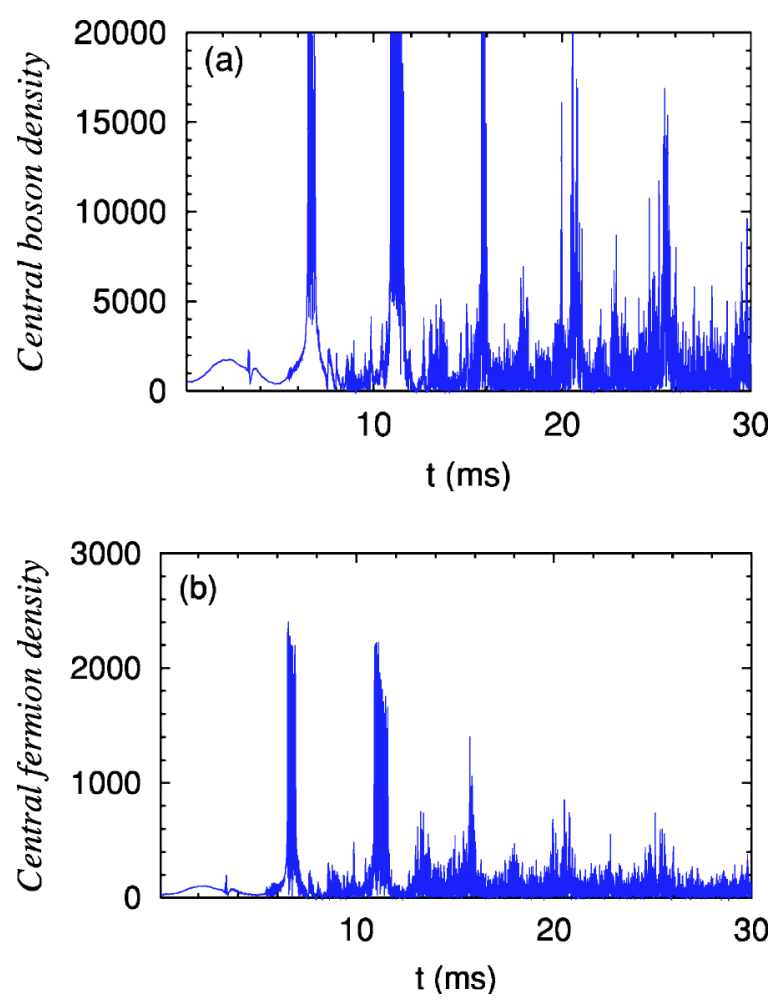

FIG. 3. Evolution of the (a) central boson probability density $\left[N_{B}\left|\psi_{B}(0, t)\right|^{2}\right]$ and (b) central fermion probability density $\left[N_{F}\left|\psi_{F}(0, t)\right|^{2}\right]$ in arbitrary units during the collapse exhibited in Figs. 1 and 2 .

fluctuation of the central density could not be due to a weak evaporation of the condensate due to recombination (2.10).

Next we consider fermionic collapse initiated by an increase of the number of bosonic atoms. In Fig. 4(a) we plot the evolution of the boson and fermion numbers in the condensate from time $t=0$ to $t=50 \mathrm{~ms}$ after the jump in the boson number from 4800 to 14400 at time $t=0$. In this case there is a decay in boson and fermion numbers. The decay of boson numbers $(\sim 2000)$ is double the decay of fermion numbers $(\sim 1000)$. To determine if this case corresponds to collapse, in Fig. 4(b) we plot the profiles of the bosonic and fermionic wave functions in arbitrary units at times $t=0$ and $t=50 \mathrm{~ms}$. At $t=50 \mathrm{~ms}$ the fermionic wave function is sharply peaked at the center and spiky in nature, whereas the reasonable smooth bosonic wave function does not exhibit any central peaking. The slowly varying final bosonic component corresponds to a weak loss of atoms and not to collapse. The collapse of the fermionic component deserves further examination. In Fig. 5 we plot the evolution of the central probability density of the fermionic $\left[N_{F}\left|\psi_{B}(0, t)\right|^{2}\right]$ component in arbitrary units during collapse exhibited in Figs. 4. The central density exhibits rapid oscillation indicating collapse. However, the fluctuation in Fig. 5 is less than those noted in Figs. 3 indicating a less violent collapse in this case. This is quite reasonable as in Fig. 2 both components undergo collapse.

The initial nonlinearities in Eqs. (2.15) and (2.16) are $\mathcal{N}_{B B}=24, \mathcal{N}_{B F}=-15, \mathcal{N}_{F B}=-60$, whereas the final nonlinearities in Figs. 1 are $\mathcal{N}_{B B}=24, \mathcal{N}_{B F}=-45, \mathcal{N}_{F B}=-180$. The final 

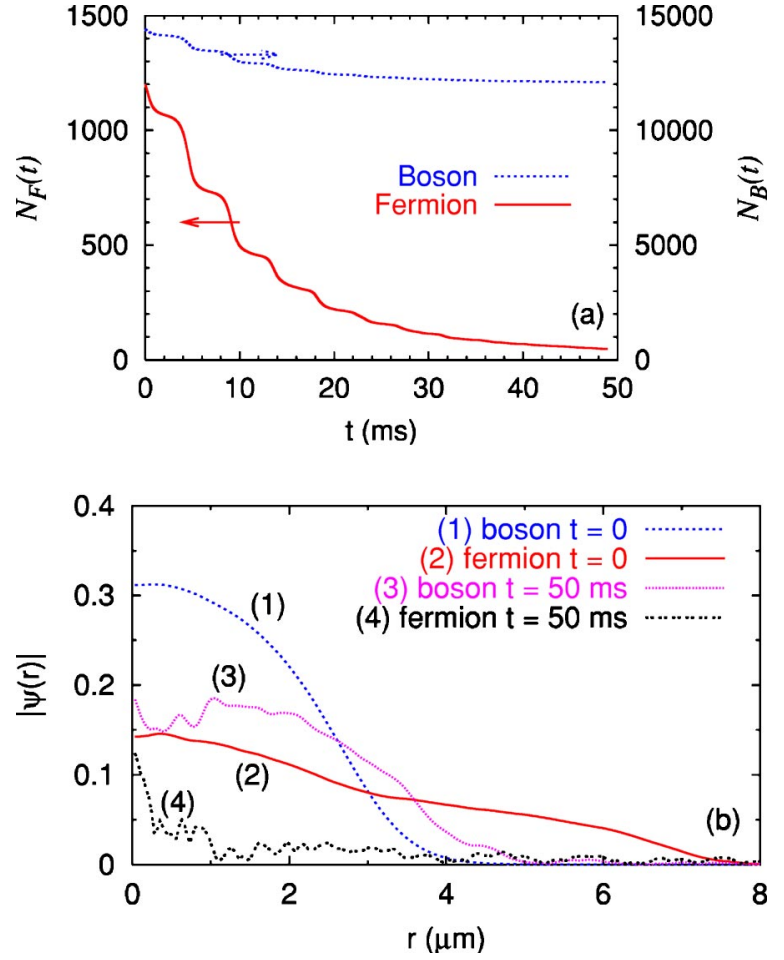

FIG. 4. (Color online) (a) The evolution of the numbers of bosons $N_{B}(t)$ and fermions $N_{F}(t)$ during collapse initiated by a jump in the boson number from 4800 to 14400 for $K_{3}=10^{-27} \mathrm{~cm}^{6} \mathrm{~s}^{-1}$. (b) The initial $(t=0)$ and final $(t=50 \mathrm{~ms})$ fermion and boson wave functions in arbitrary units before and after the collapse in (a).

attractive (negative) nonlinearities are so strong that the effective nonlinearities in both Eqs. (2.15) and (2.16) have become attractive and large in nature. This is responsible for the collapse observed in both components in Figs. 1 and 2. In the situation of Fig. 4 the final nonlinearities are $\mathcal{N}_{B B}=72$, $\mathcal{N}_{B F}=-15, \mathcal{N}_{F B}=-180$. We find that, as a result, the repulsive nonlinearity in the bosonic equation (2.15) has increased and hence the bosonic condensate does not collapse; whereas the attractive nonlinearity in the fermionic equation (2.16) has increased by a factor of 3 and hence this component undergoes collapse. However, in Fig. 4(a) there is a steady loss of atoms in the repulsive bosonic condensate during the collapse of the fermionic condensate.

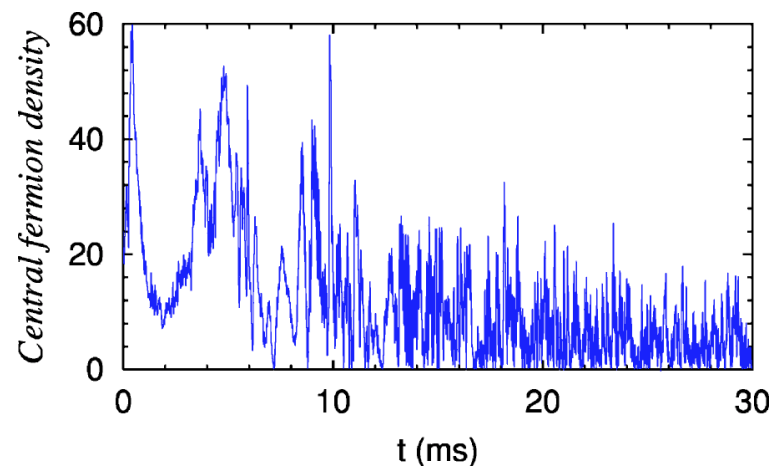

FIG. 5. Evolution of the central fermion probability density $\left[N_{F}\left|\psi_{F}(0, t)\right|^{2}\right]$ in arbitrary units during the collapse exhibited in Figs. 4.
The present study indicates that under different situations the fermionic component in a trapped condensed bosonfermion mixture can undergo a collapse due to an attractive boson-fermion interaction. In the present study we consider two situations. Of these, the fermionic collapse associated with the increase in the number of bosons has been exploited in the experiment of Modugno et al. [5]. In the present model simulation, we introduced a sudden jump in the number of atoms which is not possible in the laboratory. In the experiment of Ref. 5 a steady increase in boson numbers has been used to initiate the fermionic collapse. However, the situation of collapse exploiting a Feshbach resonance in the bosonfermion system is more exciting and controlled experiment could be done by jumping the boson-fermion scattering length $a_{B F}$ as in the collapse experiment with bosonic condensate [11]. This seems possible in ${ }^{6} \mathrm{Li}^{23} \mathrm{Na}[23]$ and ${ }^{40} \mathrm{~K}$ ${ }^{87} \mathrm{Rb}$ [24] systems using the recently discovered Feshbach resonances in these systems. The increase in the number of bosonic atoms in the boson-fermion condensate to initiate the collapse is a slow stochastic process as opposed to sudden controlled jump in the scattering length leading to a much violent collapse. In this connection we recall that the collapse of a purely bosonic ${ }^{7} \mathrm{Li}$ condensate in Ref. 10 by a steady increase of the atom number was a stochastic process, whereas the collapse of ${ }^{85} \mathrm{Rb}$ atoms in Ref. 11 by a jump in scattering length was a more violent, nevertheless more exciting, process. However, the collapse initiated by a jump in the scattering length $a_{B F}$ will require a careful study of the boson-fermion system in the search of an appropriate Feshbach resonance $[23,24]$.

An examination of Eqs. (2.15) and (2.16) with $\xi_{F}=\xi_{B}=0$ reveals that for a fixed $N_{F}$ with the increase of the boson number $N_{B}$ the attractive nonlinearity $\mathcal{N}_{F B}$ can overcome the repulsive Fermi pressure and lead to a collapse of the fermionic condensate. Hence, for a fixed $N_{B}$ and fixed values of scattering lengths, $N_{F}$ has to be larger than a critical value in order to have a stable condensate. The fermionic collapse is a function of the boson-fermion scattering length $a_{B F}$ and the boson number $N_{B}$. These aspects of collapse have been studied via equilibrium equations (2.3) and (2.4) by Modugno et al. [20]. Finally, we comment that a large number of fermions $N_{F}$ in Eqs. (2.15) and (2.16) will lead to a strong attraction in the bosonic equation (2.15) via the term $\mathcal{N}_{B F}$ which may initiate a collapse of the bosonic atoms. An increase of $N_{B}$ stabilizes the bosonic condensate but may initiate collapse in the fermionic one, whereas an increase in $N_{F}$ stabilizes the fermionic condensate but may start collapse in the bosonic one. Finally, an increase in $\left|a_{B F}\right|$ may initiate collapse in both components. A detailed study of these features in the actual experimental situation would be a welcome future work.

\section{SUMMARY}

We have suggested a coupled set of time-dependent mean-field-hydrodynamic equations for a trapped bosonfermion condensate using the (time-independent) energy functional of Ref. 9 successfully used to study the equilibrium states of the trapped boson-fermion condensate. One 
could identify collapse from the time independent formulation, where equilibrium stationary solutions for the bosonfermion mixture cease to exist. The present time-dependent generalization permits us to study nonequilibrium dynamics of the coupled boson-fermion condensate. We use the timedependent nonlinear model to study the collapse dynamics for the boson-fermion mixture. There are two possibilities for the collapse of the fermionic component. From Eq. (2.16) we see that this happens when the attractive nonlinearity $\mathcal{N}_{F B}$ becomes stronger either via an increase in boson number $N_{B}$ or via an increase in the strength of boson-fermion interaction $\left|a_{B F}\right|$. We considered both possibilities in the present numerical study. The increase of $\left|a_{B F}\right|$ leads to a collapse of both components, whereas the increase of $N_{B}$ leads to a collapse of the fermionic component alone. The collapse is more violent in the first case. The present simulation was done in a spherically symmetric model. The collapse dynamics is strongly dependent on the loss rate $K_{3}$. Once one has a good knowledge of the variation of $K_{3}$ with $\left|a_{B F}\right|$, and highquality experiment data will be available, a more realistic calculation will be worth doing in the future.

\section{ACKNOWLEDGMENTS}

The work is supported in part by the CNPq of Brazil.
[1] B. DeMarco and D. S. Jin, Science 285, 1703 (1999).

[2] K. M. O'Hara, S. L. Hemmer, M. E. Gehm, S. R. Granade, and J. E. Thomas, Science 298, 2179 (2002).

[3] F. Schreck, L. Khaykovich, K. L. Corwin, G. Ferrari, T. Bourdel, J. Cubizolles, and C. Salomon, Phys. Rev. Lett. 87, 080403 (2001); A. G. Truscott, K. E. Strecker, W. I. McAlexander, G. B. Partridge, and R. G. Hulet, Science 291, 2570 (2001).

[4] Z. Hadzibabic, C. A. Stan, K. Dieckmann, S. Gupta, M. W. Zwierlein, A. Gorlitz, and W. Ketterle, Phys. Rev. Lett. 88, 160401 (2002).

[5] G. Modugno, G. Roati, F. Riboli, F. Ferlaino, R. J. Brecha, and M. Inguscio, Science 297, 2240 (2002).

[6] G. Roati, F. Riboli, G. Modugno, and M. Inguscio, Phys. Rev. Lett. 89, 150403 (2002).

[7] K. E. Strecker, G. B. Partridge, and R. G. Hulet, Phys. Rev. Lett. 91, 080406 (2003); Z. Hadzibabic, S. Gupta, C. A. Stan, C. H. Schunck, M. W. Zwierlein, K. Dieckmann, and W. Ketterle, ibid. 91, 160401 (2003).

[8] F. Dalfovo, S. Giorgini, L. P. Pitaevskii, and S. Stringari, Rev. Mod. Phys. 71, 463 (1999).

[9] P. Capuzzi, A. Minguzzi, and M. P. Tosi, Phys. Rev. A 69, 053615 (2004); 68, 033605 (2003); 67, 053605 (2003).

[10] J. M. Gerton, D. Strekalov, I. Prodan, and R. G. Hulet, Nature (London) 408, 692 (2001).

[11] E. A. Donley, N. R. Claussen, S. L. Cornish, J. L. Roberts, E. A. Cornell, and C. E. Wieman, Nature (London) 412, 295 (2001)

[12] S. Inouye, M. R. Andrews, J. Stenger, H. J. Miesner, D. M. Stamper-Kurn, and W. Ketterle, Nature (London) 392, 151 (1998).

[13] S. K. Adhikari, Phys. Rev. A 66, 013611 (2002); 66, 043601
(2002).

[14] H. Saito and M. Ueda, Phys. Rev. A 65, 033624 (2002); L. Santos and G. V. Shlyapnikov, ibid. 68, 011602 (2002); C. M. Savage, N. P. Robins, and J. J. Hope, ibid. 67, 014304 (2003); R. A. Duine and H. T. C. Stoof, ibid. 68, 013602 (2003); E. A. Calzetta and B. L. Hu, ibid. 68, 043625 (2003); W. Bao, D. Jaksch, and P. A. Markowich, J. Phys. B 37, 329 (2004); S. K. Adhikari, ibid. 37, 1185 (2004).

[15] H. Heiselberg, C. J. Pethick, H. Smith, and L. Viverit, Phys. Rev. Lett. 85, 2418 (2000); M. J. Bijlsma, B. A. Heringa, and H. T. C. Stoof, Phys. Rev. A 61, 053601 (2000).

[16] S. K. Adhikari, Phys. Rev. A 63, 043611 (2001); S. K. Adhikari, Phys. Lett. A 281, 265 (2001).

[17] S. K. Adhikari, J. Phys. B 34, 4231 (2001).

[18] V. S. Shchesnovich, A. M. Kamchatnov, and R. A. Kraenkel, Phys. Rev. A 69, 033601 (2004).

[19] R. Roth, Phys. Rev. A 66, 013614 (2002); R. Roth and H. Feldmeier, ibid. 65, 021603 (2002); T. Miyakawa, T. Suzuki, and H. Yabu, ibid. 64, 033611 (2001); X.-J. Liu, M. Modugno, and H. Hu, ibid. 68, 053605 (2003); X.-J Liu and H. Hu, ibid. 67, 023613 (2003).

[20] M. Modugno, F. Ferlaino, F. Riboli, G. Roati, G. Modugno, and M. Inguscio, Phys. Rev. A 68, 043626 (2003).

[21] Yu. Kagan, E. L. Surkov, and G. V. Shlyapnikov, Phys. Rev. Lett. 79, 2604 (1997).

[22] S. K. Adhikari and P. Muruganandam, J. Phys. B 35, 2831 (2002); P. Muruganandam and S. K. Adhikari, ibid. 36, 2501 (2003).

[23] C. A. Stan, M. W. Zwierlein, C. H. Schunck, S. M. F. Raupach, and W. Ketterle, e-print cond-mat/0406129.

[24] S. Inouye, J. Goldwin, M. L. Olsen, C. Ticknor, J. L. Bohn, and D. S. Jin, e-print cond-mat/0406208. 